\title{
Remote Monitoring Technology for COVID-19 Patients
}

\author{
Prakash Kanade, Monis Akhtar, and Fortune David
}

\begin{abstract}
Human beings have adapted to a new way of life, from an open society to a closed world. Covid19 has brought many changes to the way people live, social distance and minimal to no contact are our goals. This could have been impossible if we were not surrounded by technology. This Perspective offers a context for the implementation of Remote Technologies, demonstrating the forms in which pandemic preparation, monitoring, research, tracking, and Health Care Technologies are effectively implemented. Our research is focused on providing a solution for handling patients at health facilities with the help of remote technologies keeping in mind the importance of social distancing and minimum to no contact to minimize the spread of the virus. The strategy is to identify the two fronts where contact with a patient is to be made. The first front is the point at which a patient needs to be detected and classified as a potential risk, at this stage the technology needs to identify the patient's symptoms, gather relevant data, and help doctors build a portfolio. The second front is where an admitted patient needs to be monitored and taken care of with regular check-ups, the technology should also take good of the patient's mental health. The paper proposes a solution to device remote technologies that could prove beneficial in the fight against the deadly coronavirus.
\end{abstract}

Index Terms - COVID-19, IoT, LeenaBOT, Robotics.

\section{INTRODUCTION}

A novel coronavirus (CoV) called '2019-nCoV' or '2019 novel coronavirus' or 'COVID-19' by the World Health Organization (WHO) is responsible for the current pneumonia outbreak that began near Wuhan City, Hubei Province, China at the beginning of December 2019 [1], [2] [3]. In some countries, the recent outbreak of COVID-19 is close to the previous outbreaks of SARS and Middle East Respiratory Syndrome (MERS) that occurred in China and Saudi Arabia in 2003 and 2012 [4], [5]. On December 31, 2019, in Wuhan City in Hubei Province central China, the China Health Authority alerted the World Health Organization (WHO) to multiple cases of pneumonia of unknown etiology [6], [7].

Since December 8, 2019, cases have been reported and several patients have worked or lived in the wholesale market for Huanan Seafood as well as other regional markets, and the compilation of comprehensive reports on the point of origin and type of wildlife species sold on the Huanan market and the destination of those animals after the market has been closed [8]. As of 1 December 2020, there were a total of 64 million cases worldwide, with a

Published on January 22, 2021.

Prakash Kanade, Hobby Researcher in Robotics, Artificial Intelligence, IOT, USA.

(e-mail: Prakashsrs@ gmail.com)

Monis Akhtar, Cambridge Institute of Technology, India.

(e-mail: monisakhtar0503@gmail.com)

Fortune David, Faculty, Facilitator, LeenaBOT Robotics, USA.

(e-mail: fortunekbz2009@gmail.com) death toll of 1.5 million people. The death rate or fatality rate in the entire world is 2.54 percent. The survival rate was 58.2 percent in the early days of the pandemic, and the mortality rate was a record high of 41.8 percent. Even if the average number of cases per day is rising, the figures are somewhat different. The survival rate has risen to 97.46 percent as of December 2020, and the mortality rate has fallen to 2.54 percent. With the spread of the pandemic, lockdowns have been introduced in almost all parts of the world, shutting down activities involving human gathering and contact, including colleges, schools, malls, churches, offices, airports, and railway stations [9]. Compared to prelockdown levels, internet services have seen consumption grow from 40 percent to 100 percent. Video conferencing services such as Zoom have seen a 10-fold rise in use [10], and content delivery services such as Akamai have seen a $30 \%$ increase in content use. A time of crisis also offers an opportunity for all technologies to look forward, adapt to new threats, and develop their capability. We assume that, regardless of COVID-19, remote technologies have immense potential for this to occur.

\section{TeChNOLOGY INVOLVED IN PRoposed SOLUTION}

In response to coronavirus, many players involved with technology came up with a variety of products that focused on making life easy and safe for everyone. [11], [12]. When it comes to healthcare there are many products in the market, however, most of them focused on providing one feature to solve any problem. There was a need for a variety of AI features that allow interactions with patients that are contextual and data-centric and provide a generic product that tackles most of the problematic scenarios that could be present in a hospital environment. The proposed solution suggests a robot build for handling patients. The technologies that would play a crucial role in building the robot are as follows

\section{A. Face Recognition}

The Robot is loaded with state-of-the-art computer vision technology that can gain an understanding of visual images or videos at a high level. Because of its contactless and noninvasive nature, the face recognition feature reduces the risk of transmitting the virus from one person to another [13]. The Robot will learn new faces and recognize them in realtime in realistic environments, which is later used to recognize the individual even under different conditions [14]. The Robot recognizes patients, identifies their age, gender, emotional levels, and computer vision does a lot more. 


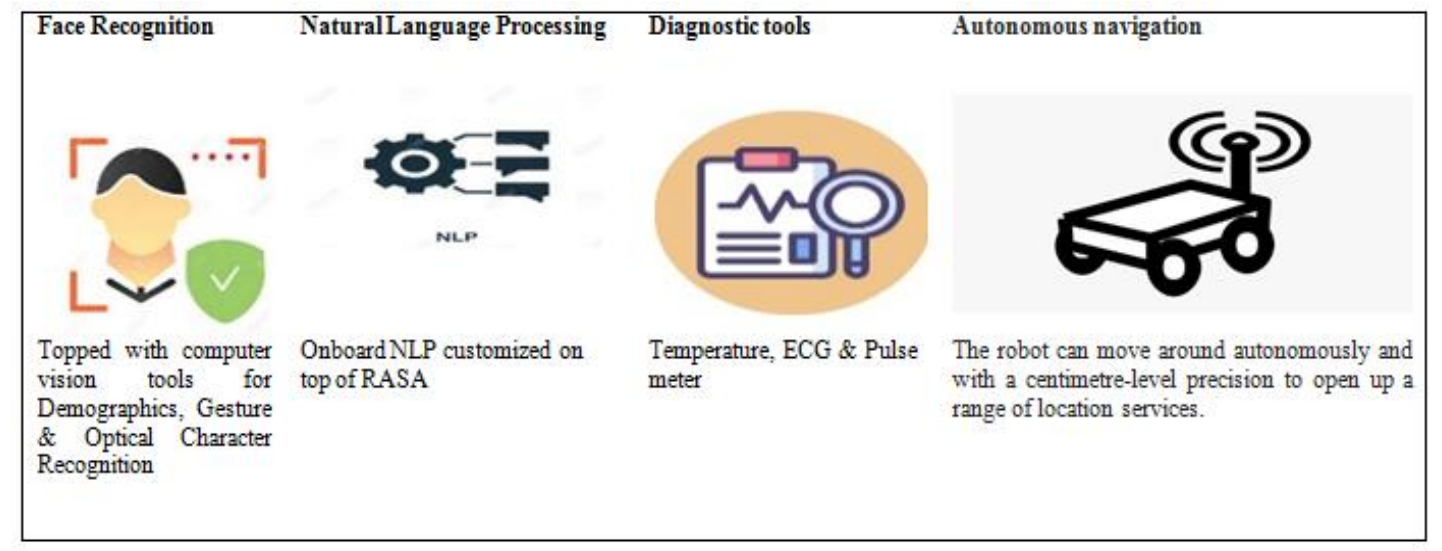

Fig. 1. Technology that can be implemented on the Robot.

\section{B. Natural Language Processing}

The Robot approaches patients with a conversation engine that will understand the user's speech input and provide appropriate material for natural language processing [15], [16]. The Robot uses RASA Open Source, which offers open-source natural language processing, to convert messages into intent [17], [18]. Centered on lowerlevel machine learning libraries such as Tensorflow and SpaCy, Rasa Open Source provides functional natural language processing software. The Robot holds substantive dialogue with the patients using NLP.

\section{Diagnostic Tools}

A thermal camera is used by The Robot to monitor the patient's temperature. Other diagnostics instruments such as the ECG and Pulse meter are also used by the robot. These results will assist doctors with the patient's diagnosis without making physical contact. This function can also be used by the doctor to examine patients on time and record patients' vital signs to monitor their condition.

\section{Autonomous Navigation}

The Robots can automate and increase production in the factory setting for a wide variety of labor-intensive tasks. To create 3D maps and provide localization, LiDARs are used [19] (which could be achieved through other means like cameras and GPS as well), but none can contend with LiDAR's efficacy.

\section{PROPOSED SOLUTION: WORKING SCENARIOS}

For various tasks that allow contactless service, the Robot can be used so that healthcare workers do not expose themselves to the virus. From patient registration, screening, seamless tele-consulting with physicians to connecting patients via video to their loved ones, the Robot performs a different role.

\section{A. Scenario I}

If a new patient enters hospital premises for a check-up, or an old patient reaches out for routine control, the first scenario occurs. The figure below illustrates how, if a patient reaches out to the hospital, the Robot will support hospitals. The Robot has a constructive discussion with the patient and sets out the goals for a contactless service that eliminates the risk to health workers of spreading the virus.

Detection: Whenever a patient goes to the hospital, the robot greets the patient with the aid of face recognition if the patient's data already exists.

Registration: If the patient database does not exist, the Robot queries the patient's name and phone number via voice and stores details in its databases, such as name, phone number, age, and gender.

Screening: The Robot does accurate thermal screening with advanced computer vision and asks relevant questions through voice.

Consulting: If the patient is showing signs of fever and other ailments, the Robot connects the patient with the doctor. The doctor after analyzing the patient's conditions, issues commands to the Robot.

Pass Issue: After getting clearances from the doctor the Robot issues a pass to the patient and directs them to the next step.

\section{B. Scenario II}

The Second scenario plays out when a patient who is admitted to the hospital comes in contact with Robot. The Robot can be used for connecting patients with their loved ones.

Its piercing eyes are equipped with facial recognition technology to help it recall people with whom it has previously interacted. A tablet attached to the Robot chest allows patients to see loved ones, as well as medical staff unable to access the wards. The Robot is mainly used by patients who are not able to communicate using their phones. The Robot is also being used for remote consultations with specialists to reduce their risk of becoming infected. Normally it is very difficult for a psychologist or a dietician to see a COVID patient, but the Robot makes it very easy [21]. There is no need for a doctor or nurse to go close to patients for reading or care in this method.

Using Bluetooth-enabled mobile devices including phones or tablets, they can do all of these at a safe distance.

When the unit is near the ward, it will automatically connect via Bluetooth to the completely networked computer system. When linked using the app installed in handheld devices, a robot hand installed on the Robot can be operated by a healthcare worker to take readings or provide medication or food. Networked Tablet mounted to the Robots chest can be used during this tough period to make a zoom call with family members to speak to loved ones. 


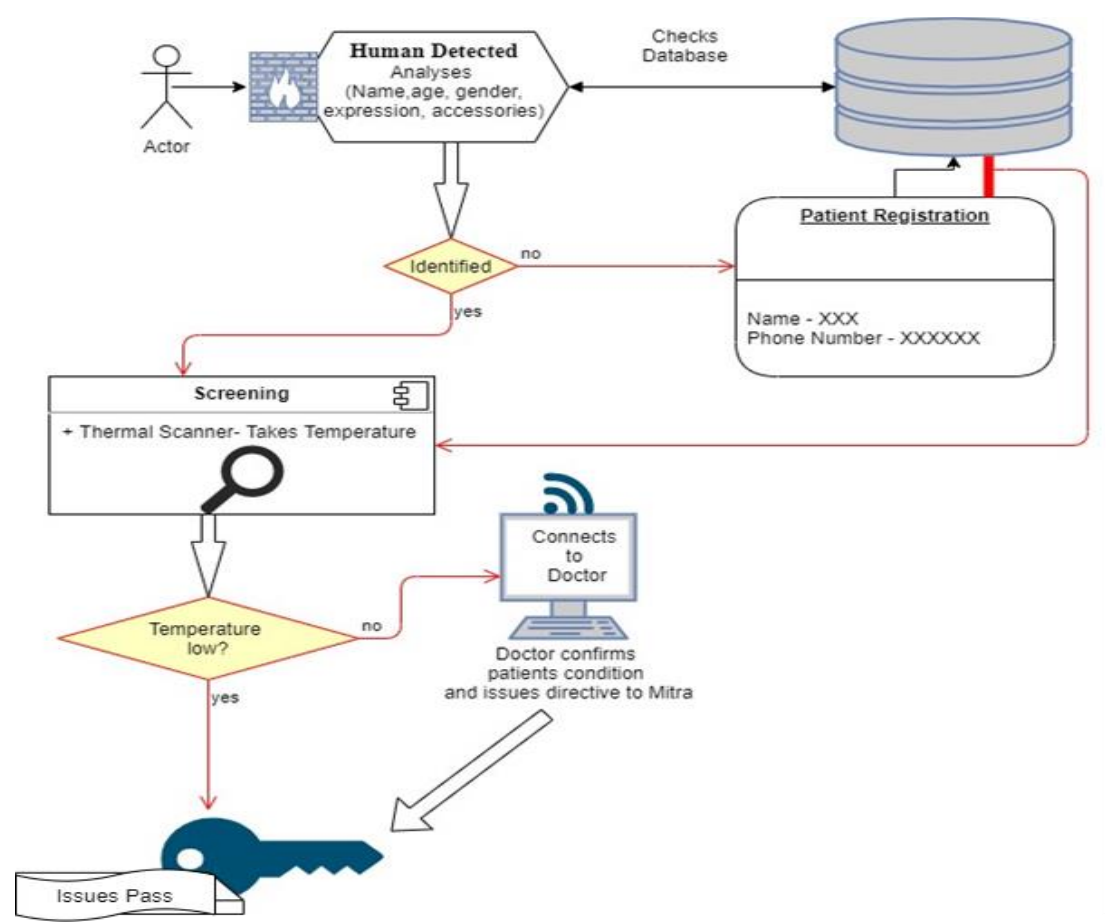

Fig. 2. Working of the Robot for patient registration and screening.

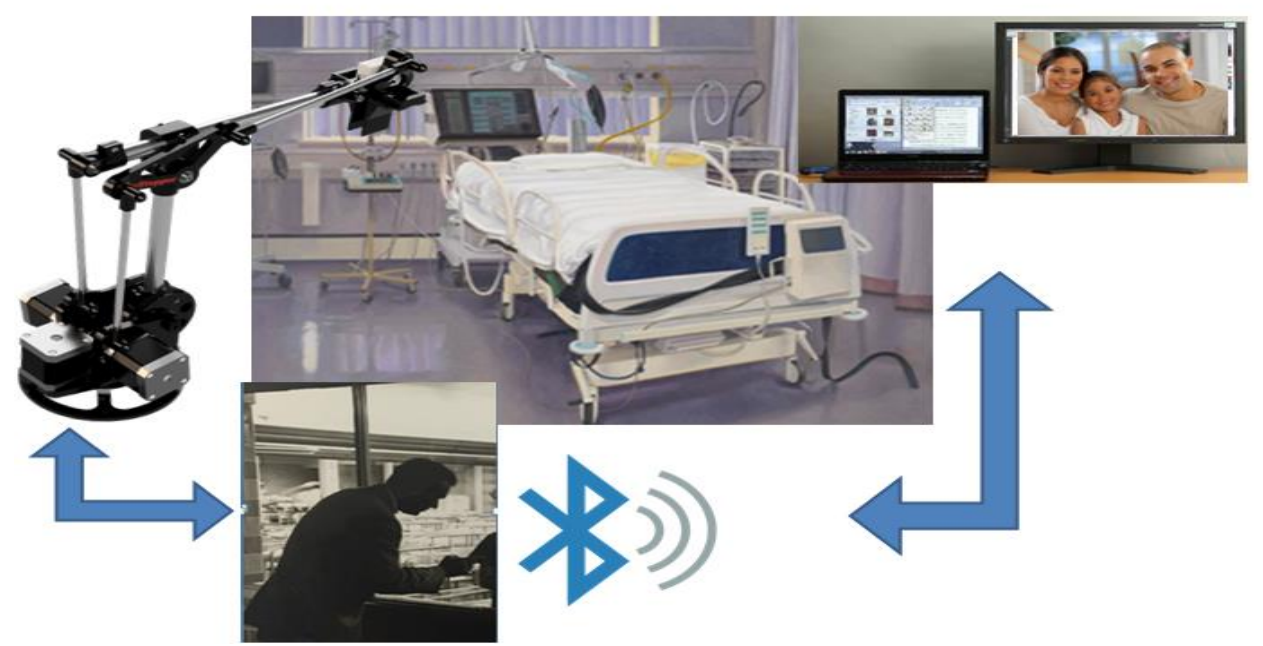

Fig. 3. Robot connects patients with their loved ones [20].

\section{CONCLUSION}

To tackle the coronavirus pandemic, researchers are exploring every possible option, and modern technology represents a captivating course. Although technological advancements have entered our day-to-day lives with numerous victories, they have also contributed to helping individuals in the very intense war against COVID-19. The papers address interest in potential work on the development of a benchmark system to test and analyze existing techniques. It can be concluded that in the war against the deadly virus technologies have a wide potential for growth, but not all of them are currently sufficiently developed to demonstrate operational impact.

\section{REFERENCES}

[1] C. e. a. Sohrabi, "World Health Organization declares global emergency: A review of the 2019 novel coronavirus (COVID-19)," International Journal of Surgery, 2020.

[2] S. P. e. a. Adhikari, "Epidemiology, causes, clinical manifestation and diagnosis, prevention and control of coronavirus disease (COVID-19) during the early outbreak period: a scoping review," Infectious diseases of poverty, vol. 9, no. 1, pp. 1-12, 2020.

[3] H. e. a. Wang, "Phase-adjusted estimation of the number of coronavirus disease 2019 cases in Wuhan, China," Cell discovery, vol. 6 , no. 1 , pp. 1-8, 2020.

[4] N. C. e. a. Peeri, "The SARS, MERS and novel coronavirus (COVID-19) epidemics, the newest and biggest global health threats: what lessons have we learned?," International journal of epidemiology, 2020.

[5] S. E. Park, "Epidemiology, virology, and clinical features of severe acute respiratory syndrome-coronavirus-2 (SARS-CoV-2; Coronavirus Disease-19)," Clinical and experimental pediatrics, vol. 63 , no. 4 , p. $119,2020$.

[6] N. e. a. Zhu, "A novel coronavirus from patients with pneumonia in China, 2019," New England Journal of Medicine, 2020. 
[7] Q. e. a. Li, "Early transmission dynamics in Wuhan, China, of novel coronavirus-infected pneumonia," New England Journal of Medicine, 2020.

[8] N. C. e. a. Peeri, "he SARS, MERS and novel coronavirus (COVID 19) epidemics, the newest and biggest global health threats: what lessons have we learned?," International journal of epidemiology, 2020.

[9] I. N. e. a. Ibeh, "Global Impacts and Nigeria Responsiveness to the COVID-19 Pandemic," International Journal of Healthcare and Medical Sciences, vol. 6, no. 4, pp. 27-45, 2020.

[10] P. D. e. a. Patel, "Rapid Development of Telehealth Capabilities within Pediatric Patient Portal Infrastructure for COVID-19 Care: Barriers, Solutions, Results.," Journal of the American Medical Informatics Association, 2020.

[11] M. e. a. Nicola, "The socio-economic implications of the coronavirus pandemic (COVID-19): A review," International journal of surgery, vol. 78, p. 185, 2020.

[12] H. a. J. K. Shin, "Reducing perceived health risk to attract hotel customers in the COVID-19 pandemic era: Focused on technology innovation for social distancing and cleanliness," International Journal of Hospitality Management, vol. 91, p. 102664, 2020.

[13] A. C. S. a. K. I.-K. W. Prati, "Sensors, vision and networks: From video surveillance to activity recognition and health monitoring," Journal of Ambient Intelligence and Smart Environments , vol. 11, no. 1, pp. 5-22, 2019.

[14] C. L. E. S. a. E. F. M. Cruz, "Real-time face recognition for human robot interaction," IEEE International Conference on Automatic Face \& Gesture Recognition, vol. 8, pp. 1-6, 2008.

[15] J. a. C. D. M. Hirschberg, "Advances in natural language processing," Science, vol. 349, no. 6245, pp. 261-266, 2015.

[16] S. A. a. J. C. W. Abdul-Kader, "Survey on chatbot design techniques in speech conversation systems," International Journal of Advanced Computer Science and Applications, vol. 6, no. 7, 2015.

[17] A. K. R. a. S. S. Singh, "Building an enterprise chatbot: Work with protected enterprise data using open source frameworks," Apress, 2019.

[18] A. K. R. a. S. S. Singh, "Introduction to Microsoft Bot, RASA, and Google Dialogflow," Apress, pp. 281-302, 2019.

[19] W. G. P. a. P. N. Maddern, "Leveraging experience for large-scale LIDAR localisation in changing cities," IEEE International Conference on Robotics and Automation (ICRA), 2015.

[20] P. Kanade, M. Akhtar and F. David, "Computer Networking and Technology Improvement in the Age of COVID-19," International Journal of Advanced Networking and Applications (IJANA), vol. 12, no. 03, pp. 4592-4595(2020), 2020.

[21] P. Kanade and S. Kanade, "Medical Assistant Robot ARM for COVID-19 Patients Treatment," International Research Journal of Engineering and Technology (IRJET), vol. 7, no. 10, p. 10.5281/zenodo.4393125, 2020. 